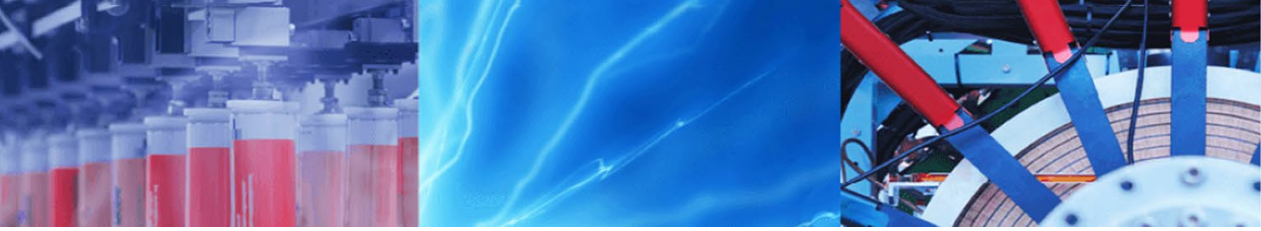

Research Article

\title{
Mechanical properties of PTT fibers by sustainable horizontal isothermal bath process
}

\author{
Mesbah Najafi ${ }^{1} \cdot$ Richard Kotek $^{1}$
}

(c) Springer Nature Switzerland AG 2019

\begin{abstract}
The melt spinning process of poly (trimethylene terephthalate) was modified by the inclusion of horizontal isothermal bath (HIB) in the extrusion line. Several parameters including liquid depth, liquid temperature, and take-up speed were utilized in this study. The structural and mechanical properties of the fibers were characterized by DSC, X-ray, SEM, optical birefringence, and tensile testing. The results showed that the bath treatment increased considerably the fiber birefringence up to 0.066 by $66 \%$ and increased the crystallinity up to $47.94 \%$ by $100 \%$ compared to the control (no HIB) fibers. Drawing the HIB fibers with a low draw ratio (DR) of 1.11 increased the tensile strength up to $4.76 \mathrm{~g} / \mathrm{d}$ and reduced the tensile strain down to $51.76 \%$. Such strength is greater than the maximum value of $3.3 \mathrm{~g} / \mathrm{d}$ reported before. The obtained results can widen application of PTT fibers in technical woven/nonwoven fiber products.
\end{abstract}

Keywords Poly(trimethylene terephthalate) $\cdot$ Horizontal isothermal bath (HIB) $\cdot$ Melt spinning process · Fiber tensile strength · Compact fibrillar morphology · Molecular orientation

\section{Introduction}

Poly(trimethylene terephthalate) (PTT) also known as 3GT is an aromatic polyester made from polycondensation of 1,3-propanediol (PDO) and terephthalic acid or dimethyl terephthalate. The chemical structure of this polyester is shown in Fig. 1. Although its unique properties have been known for many years, the polymer was not commercially available until recent years due to high production cost of PDO [1]. PTT has combined physical properties of nylons (PA-6 \& PA-66) and other aromatic polyesters (PET \& PBT) and thus is an interesting polymer for various applications $[2,3]$. In fact, high resiliency, superior elastic recovery, high bulk, and soft hand make this polymer attractive for engineering textile fibers, carpets, fabrics, nonwovens, films and plastics. PTT filaments are the most promising candidate for replacement of PET fibers, due to the properties such as lower melting point, easier processing and dyeing and higher flexibility [1, 4-6].
Nevertheless, the low tensile performance of PTT filaments limits their wide applications in industrial products. Several studies have been done on modifying mechanical characteristics of melt-spun PTT fibers [1, 4-8]. Wu et al. [9] examined the effect of take-up speed on the tensile properties of PTT filaments. They reported the low tenacity and modulus of 3.3 and $22 \mathrm{~g} / \mathrm{d}$ for a very high wind-up speed of $8000 \mathrm{~m} / \mathrm{min}$. Also, Lyoo et al. [4] examined the influence of zone-drawing on the tensile performance of PTT fiber. Draw ratio (DR) of 2.5 on PTT fiber produced at $1000 \mathrm{rpm}$ increased the tenacity and modulus only up to $0.3 \mathrm{GPa}$ $(2.56 \mathrm{~g} / \mathrm{d})$ and $1.2 \mathrm{GPa}(9.83 \mathrm{~g} / \mathrm{d})$, respectively. Moreover, Kim et al. [1] compared the tensile and structural properties of PTT and PET fibers at various take-up speeds. They found that the spinning speed is less effective in improving the crystal formation, molecular orientation, and tensile strength of PTT fibers. The reason is related to the additional methylene group in the PTT unit structure. Compared to PET, aromatic polyesters such as PTT and

Richard Kotek, rkotek@ncsu.edu| ${ }^{1}$ Textile Engineering Chemistry and Science Department, Wilson College of Textiles, North Carolina State University, Raleigh, NC 27606, USA.

SN Applied Sciences (2019) 1:1191 | https://doi.org/10.1007/s42452-019-1198-5

Received: 10 May 2019 / Accepted: 28 August 2019 / Published online: 10 September 2019 


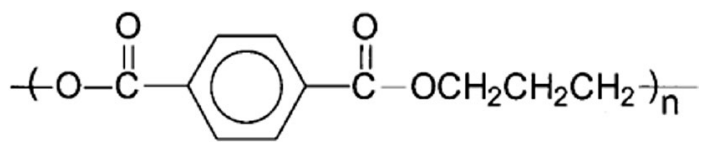

Fig. 1 Chemical structure of PTT [3]

PBT have additional methylene group(s) in their structure which make them to have more flexible polymer chains and faster crystallization rate than PET (Fig. 2) [1, 9]. The rapid crystal formation during spinning process can limit the chain mobility required for more orientation/crystallization which affects the tensile performance of PTT fibers.

To address this problem, the chain mobility and the crystal formation of the PTT chains need to be controlled better during spinning process. This can be achieved by using horizontal isothermal bath (HIB) method. HIB is a modified version of melt spinning process in which a liquid bath is used horizontally between the spinneret and the winder (Fig. 3). The bath acts like a drawing tool and provides several additional controllable processing parameters for better tension, time, and temperature control of the polymer chains in the spinning line. The liquid temperature is usually set $30-40^{\circ} \mathrm{C}$ higher than polymer glass transition temperature $\left(\mathrm{T}_{\mathrm{g}}\right)$ to provide enough mobility for molecular chains to become more oriented and crystallized inside the fibers. The liquid can contribute to higher tensile properties in two different ways: First, it provides high frictional drag on the runner filaments inside the bath. Second, it increases the mobility of amorphous chains for more molecular orientation and crystal formation. The liquid bath treatment has already been applied to several semi-crystalline polymers (e.g. PP [11, 12], PEN

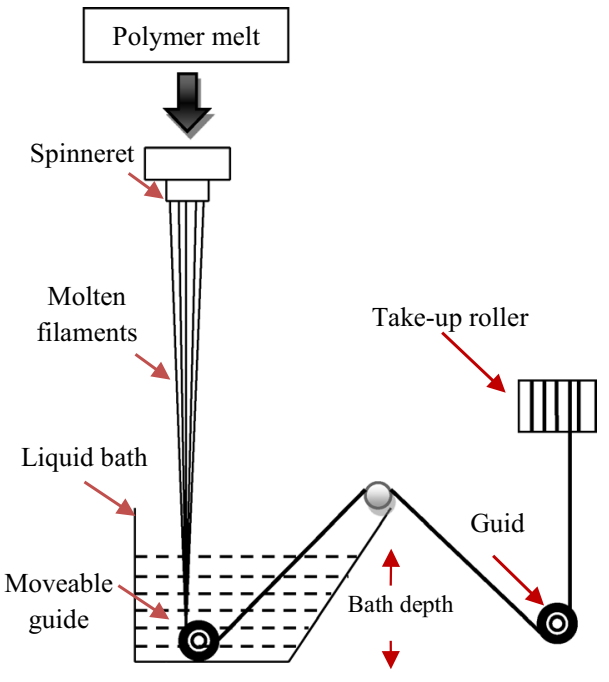

Fig. 3 Scheme of horizontal isothermal bath (HIB) method [25]

$[13,14]$, PET [15-21], PA-6 [22-24]), and fibers with tensile properties comparable to and even better than melt-spun yarns were produced.

The purpose of this study is to examine the effect of HIB process on the tensile properties of PTT fibers. This is the first time that such eco-friendly method is applied to this polyester. Simplicity, commercial availability, and cost effectiveness are some of the important features of this sustainable method. To achieve that, two types of samples including control (no HIB) and HIB were made and their structure-property relationship were characterized, compared, and analyzed using tensile testing, DSC, X-ray, SEM, and optical birefringence tests. In addition, the control and HIB samples were drawn with a low draw ratio to

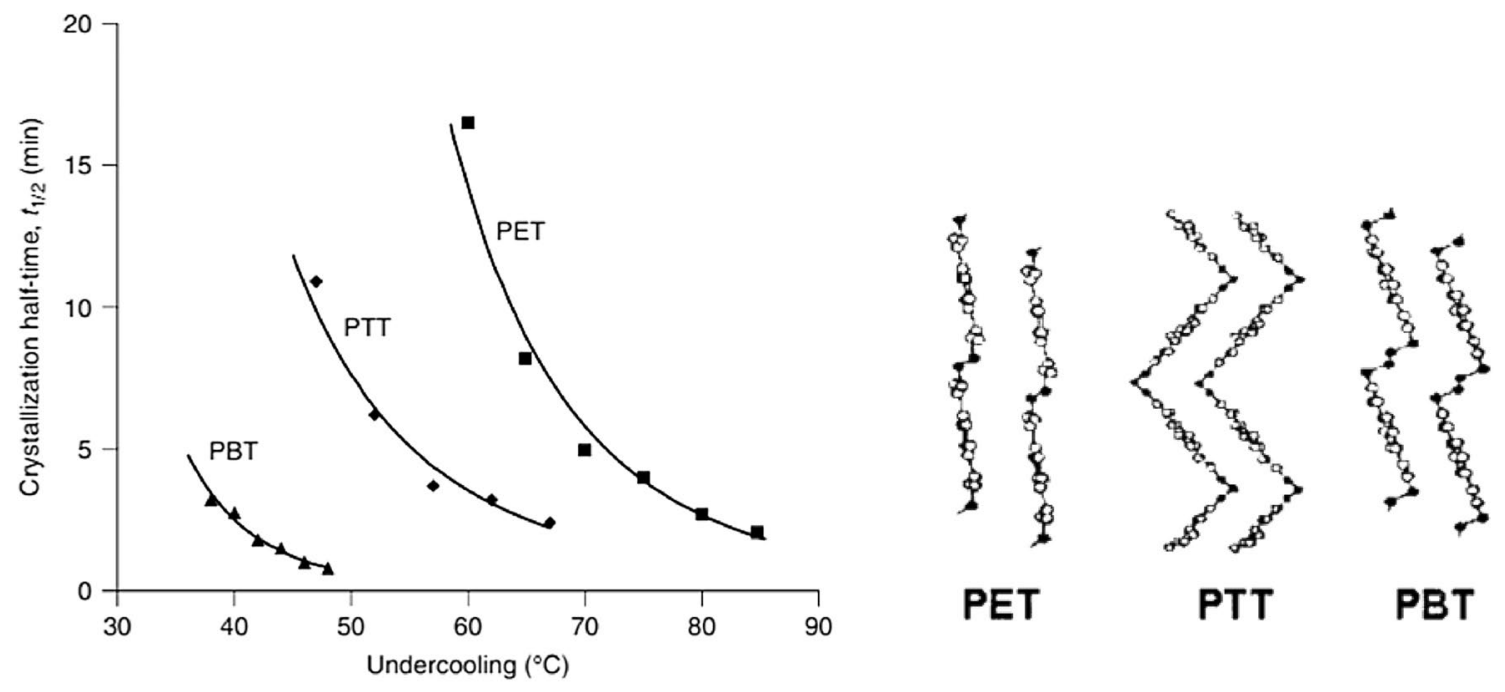

Fig. 2 Left: comparison of crystallization half-times $\left(t_{1 / 2}\right)$ for PET, PTT, and PBT at the same under-cooling degrees from the melts [10]. Right: polymer structures of three polyesters: PET, PTT and PBT, the methylene groups are shown as black filled circles [4] 
examine the effect of drawing on the tensile performance of the fibers.

\section{Experimental approach}

\subsection{Materials and sample preparation}

The PTT chips with intrinsic viscosity of $0.97 \mathrm{dl} / \mathrm{g}$ were provided from Shell Chemical Company. The viscosity-average molecular weight was estimated to be about 75,500 $\mathrm{D}$ [26]. The polymer chips were vacuum dried at $140{ }^{\circ} \mathrm{C}$ for $12 \mathrm{~h}$ before use. Alex James machine equipped with 8-hole spinneret with exit diameter of $0.3 \mathrm{~mm}$ was used for melt extrusion and spinning. The extrusion temperature was set at $265^{\circ}$, and the take-up speed was in the range of $1000-2500 \mathrm{~m} / \mathrm{min}$ for both untreated (no HIB) and treated (HIB) yarns. Water was used as the liquid medium, since it is eco-friendly and inexpensive. Various liquid temperature $\left(25-88^{\circ} \mathrm{C}\right)$ and liquid depth $(9-18 \mathrm{~cm})$ were used for making the HIB yarns (Fig. 3). After melt-spinning, the asspun untreated and treated yarns were drawn at a draw ratio close to 1.11 (which was the maximum DR for HIB filaments) by using a modified tensile tester. The draw temperature and speed were set to $70^{\circ} \mathrm{C}$ and $50 \mathrm{~mm} / \mathrm{min}$, respectively.

\subsection{Experimental techniques}

Denier measurement and tensile testing were performed on the individual PTT filaments. The denier was measured using a Vibromat ME tester. Tenacity, modulus, and strain were determined using a MTS Q-test/5 universal testing machine according to ASTM D3822. A 5-pound load cell, a gauge length of $25.4 \mathrm{~mm}$, and a constant crosshead speed of $15 \mathrm{~mm} / \mathrm{min}$ were used for the tensile testing of all samples.

The thermal traces of the yarns were investigated by Differential Scanning Calorimetry (DSC) measurement using a Perkin-Elmer Diamond DSC Model 7. 3-5 mg of each sample was encapsulated in a nonvolatile aluminum pan and then heated at the heating rate of $20^{\circ} \mathrm{C} / \mathrm{min}$ to $250^{\circ} \mathrm{C}$. The degree of crystallinity of the fibers was calculated from Eq. 1:

Degree of crystallinity $(\%)=\frac{\left(\Delta \mathrm{H}_{\mathrm{m}}-\Delta \mathrm{H}_{\mathrm{c}}\right) * 100}{\Delta \mathrm{H}^{\circ}}$

where $\Delta \mathrm{H}_{\mathrm{m}}$ and $\Delta \mathrm{H}_{\mathrm{c}}$ are the crystalline heat of fusion and the exotherm of cold crystallization respectively, and $\Delta H^{\circ}$ is the heat of fusion of perfect crystals of PTT $30 \pm 2 \mathrm{~kJ} / \mathrm{mol}$ $(135.9 \mathrm{~J} / \mathrm{g})$ [9].
XRD scans were obtained on Bruker AXS General Area Detector Diffraction System (GADDS) using CuKa radiation source, $\lambda=1.542 \AA$, generated at $30 \mathrm{kV}$ and $30 \mathrm{~mA}$. The data was measured in transmission geometry. The data points were integrated by GADDS software from $2 \theta$ angle in the range of $5^{\circ}-40^{\circ}$ and step wise of $0.1^{\circ} \mathrm{A}$. Equatorial scan $(\phi=0)$ was used to determine crystallinity, crystal type, and crystal size of the samples.

The apparent crystal sizes (ACS) were calculated from the Scherrer equation:

$\mathrm{ACS}=\frac{0.9 \lambda}{(\Delta 2 \theta) \cos \theta}$

where $\theta$ is the half value of the peak position, $\lambda$ is the $X$-ray wavelength, and $(\Delta 2 \theta)$ is the full-width at half-maximum (FWHM) of the crystalline peak in radians.

The refractive indices of the filaments were measured by a Nikon polarizing microscope using a series of mineral oil refractive index liquids. The birefringence $(\Delta n)$ was calculated from the difference of the perpendicular (n! $)$ and the parallel $(n \|)$ refractive indices as follows:

$\Delta \mathrm{n}=\mathrm{n} \|-\mathrm{n} \underline{1}$

\section{Results and discussions}

\subsection{Tensile properties}

\subsubsection{Effect of HIB treatment}

Table 1 displays the tensile testing results of as-spun control and HIB PTT fibers. As can be seen, the HIB samples have higher tenacity and lower strain than the control samples. For example, at $2000 \mathrm{~m} / \mathrm{min}$ the HIB treatment increased the tensile strength from 1.79 to $4.04 \mathrm{~g} / \mathrm{d}$ by ca. $125.7 \%$ and reduce considerably the tensile strain from 233.65 to $85.09 \%$. The highest obtained tenacity for HIB filaments was $4.04 \mathrm{~g} / \mathrm{d}$ which are related to the take-up speed of $2000 \mathrm{~m} / \mathrm{min}$, and the liquid depth and temperature of $18 \mathrm{~cm}$, and $88^{\circ} \mathrm{C}$, respectively. Such superior tensile strength is greater than the maximum value $(3.3 \mathrm{~g} / \mathrm{d})$ reported before [1]. It is surprising that such superior tenacity is obtained at a low take up speed $(2000 \mathrm{~m} / \mathrm{min}$ ) and simply through entering the threadline into the bath for just some milliseconds, which otherwise needs much higher take-up speeds $(7000-8000 \mathrm{~m} / \mathrm{min})$ in traditional melt spinning process. As mentioned, the HIB process acts like a drawing tool during melt spinning. When the yarn enters into the bath, the high liquid depth and take-up speed result in a high frictional drag on the running filaments. Also, the high liquid temperature provides enough mobility for the amorphous chains to get oriented/crystallized under the liquid drag, which leads the HIB fibers 
Table 1 Tensile properties of as-spun control (no HIB) and HIB PTT fibers

\begin{tabular}{llllllr}
\hline Sample ID & $\begin{array}{l}\text { Take up } \\
\text { speed }(\mathrm{m} / \\
\text { min) }\end{array}$ & $\begin{array}{l}\text { Liquid } \\
\text { depth } \\
(\mathrm{cm})\end{array}$ & $\begin{array}{l}\text { Liquid } \\
\text { temp. (C) }\end{array}$ & Tenacity (g/d) & Modulus (g/d) & Strain (\%) \\
\hline UC-1000 & 1000 & - & - & $1.14 \pm 0.06$ & $12.56 \pm 1.09$ & $521 \pm 55.39$ \\
UC-2000 & 2000 & - & - & $1.79 \pm 0.19$ & $16.72 \pm 1.03$ & $233.65 \pm 24.62$ \\
UC-2500 & 2500 & - & - & $2.19 \pm 0.12$ & $18.81 \pm 1.39$ & $169.06 \pm 15.33$ \\
UH1-1000 & 1000 & 18 & 55 & $2.5 \pm 0.09$ & $20.69 \pm 1.03$ & $149.27 \pm 13.67$ \\
UH2-1000 & 1000 & 18 & 88 & $3.17 \pm 0.13$ & $17.21 \pm 0.51$ & $147.54 \pm 18.20$ \\
UH1-2000 & 2000 & 9.3 & 25 & $2.19 \pm 0.12$ & $21.22 \pm 0.93$ & $129.12 \pm 10.12$ \\
UH2-2000 & 2000 & 9.3 & 88 & $2.73 \pm 0.08$ & $21.80 \pm 0.70$ & $98.53 \pm 5.39$ \\
UH3-2000 & 2000 & 18 & 55 & $3.33 \pm 0.10$ & $18.06 \pm 0.51$ & $89.97 \pm 7.18$ \\
UH4-2000 & 2000 & 18 & 88 & $4.04 \pm 0.23$ & $18.02 \pm 0.88$ & $85.09 \pm 6.25$ \\
UH1-2500 & 2500 & 18 & 88 & $4.06 \pm 0.16$ & $18.33 \pm 0.71$ & $80.99 \pm 6.99$ \\
\hline
\end{tabular}

UC undrawn control, UH undrawn HIB to have superior tensile properties than the conventional melt-spun fibers.

The tensile results of HIB filaments at different conditions of the bath treatment are also shown in Table 1. At the same take-up/liquid depth, tenacity increased and the strain decreased with the increasing liquid temperature. A higher tensile strength was obtained when the liquid temperature increased over the polymer $\mathrm{T}_{\mathrm{g}}$. Similar observations were reported for the effect of liquid temperature in former HIB studies on PET fibers $[21,25]$. This can be explained by the fact that at the temperatures higher than the $\mathrm{T}_{\mathrm{g}}$, the polymer chains in the amorphous region are in the mobile molten state, and they can become more oriented/crystallized in the fiber under the high drag of the bath, and thus higher tenacity can be achieved. The influences of liquid depth and take-up speed on fiber tensile performance are also shown in Table 1. The higher values of the both parameters increased the drag force on the filaments and resulted in higher tenacity and lower strain. Nevertheless, when the take-up speed increased up to $2500 \mathrm{~m} / \mathrm{min}$, the tensile strength remained almost constant. The reason for this is that when very high threadline tension is induced on the filaments by the higher speed, the polymer chains renders less mobile and the crystal formation and the molecular orientation may be restricted and this prevents further increase in the tensile properties.

Table 1 also compares the initial modulus of PTT fibers before and after the bath treatment. As it can be observed, the HIB samples have higher tensile modulus than the control samples at a certain take-up speed. The highest obtained modulus is about $22.5 \mathrm{~g} / \mathrm{d}$ which is comparable to the maximum value reported before for melt spun PTT fibers $[1,9]$. We could not see any specific trends for the effect of liquid drag or threadline tension on the fiber modulus. Such behavior is different from that of PET fibers where the initial modulus increases with the threadline stress. Kim et al. [1] examined the effect of spinning speed on the tensile properties of melt-spun PET and PTT fibers. They found that the initial modulus of PTT fibers remained almost constant with wind-up speed, whereas the modulus of the PET fibers increased remarkably with the takeup speed. Also, Avci et al. [25] studied the effect of HIB treatment on the tensile properties of PET filaments. They reported that the initial modulus of the polyester fibers increased considerably after HIB treatment.

The low modulus of PTT fibers in HIB and spinning processes can be explained by its polymer structure. Compared to PET, PTT has an additional methylene group in its backbone structure which gives this polyester a special conformation. In fact, the trimethylene glycol segment $\left(-\mathrm{CH}_{2}-\mathrm{CH}_{2}-\mathrm{CH}_{2}-\right)$ of PTT has highly contracted low energy trans-gauche-gauche-trans (tggt) conformation, whereas the ethylene glycol segment $\left(-\mathrm{CH}_{2}-\mathrm{CH}_{2}-\right)$ of PET has high energy trans-trans-trans (ttt) conformation in its crystal [27]. The gauche conformers in PTT are very compliant and give this polyester a helical structure with much lower crystal modulus (2.59 GPa) than PET (107 GPa) [28]. Also, the amorphous phase in PTT has a low modulus close to that of the crystalline region [9]. Thus, the extra methylene $\left(-\mathrm{CH}_{2}-\right)$ segment in the PTT unit results in a flexible helical structure with a low crystal, amorphous, and fiber modulus.

\subsubsection{Effect of drawing}

The treated and untreated samples with the best tensile strength were drawn with a modified tensile tester equipped with a heating tower as shown in Fig. 4. The maximum extension for the HIB yarn was observed at the temperature of $70{ }^{\circ} \mathrm{C}$. The draw ratio (DR) was calculated as the ratio of the length of the yarn before and after the drawing. Both untreated and treated yarns were drawn 


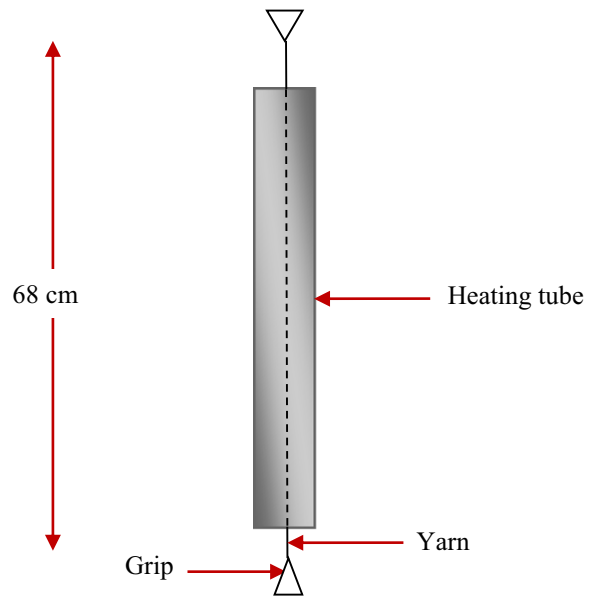

Fig. 4 Modified tensile tester equipped with a heating tower for drawing PTT yarns

with the same extension ( $405 \mathrm{~mm}$ ) with no filament breakage. The draw ratios for the control and HIB yarns after elastic recovery were at 1.40 and 1.11 , respectively. Table 2 compares the tensile properties of control and HIB fibers before and after drawing. As can be seen from this table, drawing increased the tensile strength and reduced the tensile strain of the samples. For the HIB fiber, the DR of 1.11 raised the tenacity from $4.04 \pm 0.23$ to $4.76 \pm 0.28 \mathrm{~g} / \mathrm{d}$ by $17.8 \%$ and reduced the strain at break from $85.09 \pm 6.25$ down to $51.76 \pm 5.32 \%$ by $39.2 \%$. As it will be shown later, such high tenacity is due to the crystal formation and molecular orientation occurred during the drawing.

Figure 5 displays the stress-strain curve of undrawn and drawn control and HIB PTT filaments at $2000 \mathrm{~m} / \mathrm{min}$. As this figure shows the behavior of the samples to stress and deformation is completely different. The undrawn control fibers (UC-1000 and UC-2000) have much longer yielding region than the HIB filaments (UH2-1000 and UH4-2000). The length of the yielding plateau is related to sliding of the amorphous chains in the fibers during elongation [1]. Thus, the shorter yielding of HIB samples indicates the amorphous chains have lower mobility because of the higher orientation in non-crystalline region. When the

Table 2 Tensile properties of undrawn and drawn control and HIB PTT fibers

\begin{tabular}{llll}
\hline Sample type & Tenacity $(\mathrm{g} / \mathrm{d})$ & Modulus $(\mathrm{g} / \mathrm{d})$ & Strain $(\%)$ \\
\hline UC-2000 & $1.79 \pm 0.19$ & $16.72 \pm 1.03$ & $233.65 \pm 24.62$ \\
DC-2000 & $2.82 \pm 0.33$ & $21.28 \pm 1.76$ & $100.87 \pm 11.14$ \\
UH4-2000 & $4.04 \pm 0.23$ & $18.02 \pm 0.88$ & $85.09 \pm 6.25$ \\
DH4-2000 & $4.76 \pm 0.28$ & $17.59 \pm 1.60$ & $51.76 \pm 5.32$ \\
\hline
\end{tabular}

UC Undrawn control, DC Drawn control $(\mathrm{DR}=1.40), \mathrm{UH}$ Undrawn $\mathrm{HIB}, \mathrm{DH}$ Drawn HIB (DR=1.11)

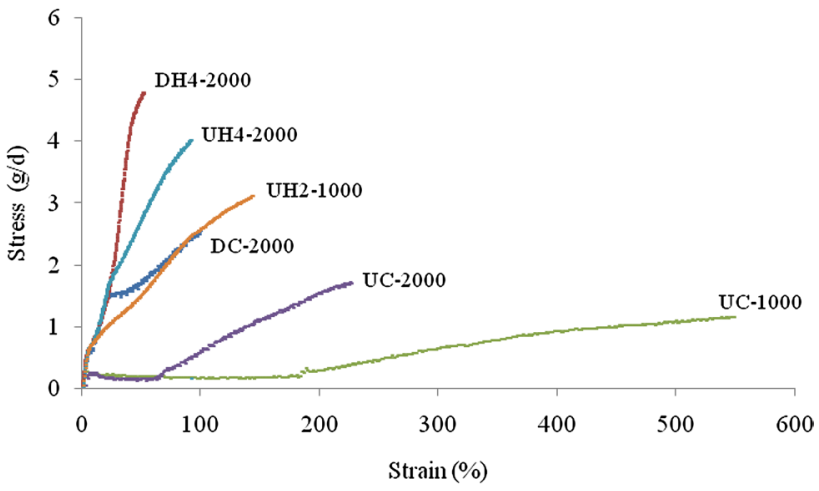

Fig. 5 Stress-strain curve of undrawn and drawn HIB and control PTT fibers

polyester filaments enter into the bath, the high liquid temperature increases the mobility of amorphous chains which under the high frictional drag of the liquid results in high molecular orientation and crystal formation and thus higher tenacity and lower elongation/yielding can be obtained, making these fibers a promising material for technical textile applications.

\subsection{Thermal properties}

Figure 6 shows the thermograms of undrawn HIB and control PTT yarns at different take-up speeds. As can be seen, the shape of the melting peaks (at $225^{\circ} \mathrm{C}$ ) in the control samples is different from that of the HIB samples. The melting thermogram of the control yarns has less symmetrical shape, implying the wider crystal size and perfection in those samples. However, when PTT fibers experienced the HIB treatment, the melting peak becomes more symmetrical. In addition, Fig. 6 shows the control fibers have a cold crystallization peak (around 64 and $73^{\circ} \mathrm{C}$ ), the area and temperature of which are decreased with the spinning speed. Such cold crystallization disappears completely in the thermograph after liquid bath treatment on the yarn. The crystal formation above $T_{g}$ in the DSC is related to chain mobility and molecular orientation in the amorphous region. At the higher orientation, the amorphous chain has lower mobility and there is lower possibility for crystal formation above the $\mathrm{T}_{\mathrm{g}}$. Based on this, the elimination of cold crystallization in the thermogram of HIB samples indicates higher molecular orientation in their non-crystalline region.

Table 3 displays the thermal properties of the undrawn and drawn control and HIB samples at take-up speeds of 1000 and $2000 \mathrm{~m} / \mathrm{min}$. The undrawn control samples have cold crystallization temperatures $\left(T_{c}\right)$ at 73.46 and $65.3^{\circ} \mathrm{C}$. The UC-2000 has lower $\mathrm{T}_{\mathrm{C}}$ than UC-1000 indicating lower mobility and higher orientation of the amorphous 


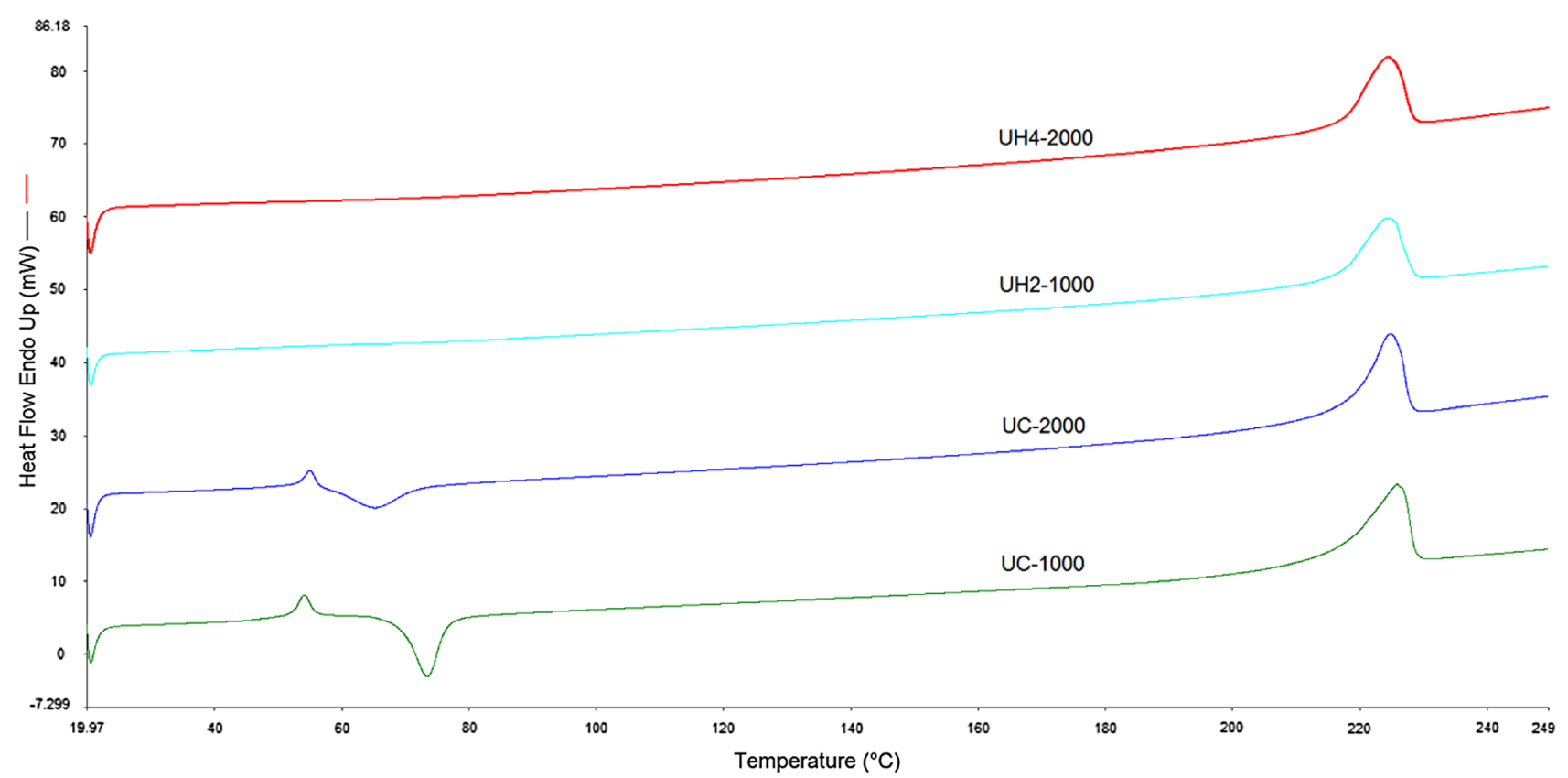

Fig. 6 DSC curves of undrawn HIB and control PTT yarns at 1000 and 2000 m/min take-up speeds

Table 3 Thermal properties of undrawn and drawn control and HIB PTT yarns

\begin{tabular}{|c|c|c|c|c|c|c|c|}
\hline \multirow[t]{2}{*}{ Sample type } & \multirow{2}{*}{$\begin{array}{l}\text { Spinning condi- } \\
\text { tions }\left(\mathrm{cm},{ }^{\circ} \mathrm{C}, \mathrm{m} /\right. \\
\text { min) }\end{array}$} & \multirow[t]{2}{*}{$\mathrm{T}_{\mathrm{c}}\left({ }^{\circ} \mathrm{C}\right)$} & \multirow[t]{2}{*}{$\mathrm{T}_{\mathrm{m}}\left({ }^{\circ} \mathrm{C}\right)$} & & \multicolumn{2}{|l|}{$\Delta \mathrm{H}(\mathrm{J} / \mathrm{g})$} & \multirow{2}{*}{$\begin{array}{l}\text { Degree of } \\
\text { crystallinity } \\
\text { (\%) }\end{array}$} \\
\hline & & & & & Crystallization & Melting & \\
\hline UC-1000 &,,-- 1000 & 73.46 & 0 & 225.83 & 31.741 & 56.069 & 17.90 \\
\hline UC-2000 &,,-- 2000 & 65.3 & - & 224.7 & 23.456 & 55.958 & 23.92 \\
\hline UH-1000 & $88,18,1000$ & - & - & 223.33 & - & 64.69 & 47.60 \\
\hline UH4-2000 & $88,18,2000$ & - & - & 222.96 & - & 65.15 & 47.94 \\
\hline DC-2000 &,,-- 2000 & - & - & 223.93 & - & 61.047 & 44.92 \\
\hline DH4-2000 & $88,18,2000$ & - & 218.36 & 222.25 & - & 68.045 & 50.06 \\
\hline
\end{tabular}

chains in that sample. The melting temperature $\left(T_{m}\right)$ was observed at $222-226^{\circ} \mathrm{C}$ for the control and HIB samples. No considerable changes were observed in $\mathrm{T}_{\mathrm{m}}$ before and after HIB treatment. Table 3 also compares the degree of crystallinity $(X)$ of the undrawn and drawn PTT yarns. For the control sample, the higher take-up speed increased the crystallinity from 17.90 to $23.92 \%$ due to stress induced crystallization. Such observation is in agreement with the findings of former studies $[1,9,26]$. Moreover, the table reveals that as-spun HIB samples have much higher crystallinity than the as-spun control samples. For example, at $2000 \mathrm{~m} / \mathrm{min}$ the crystallinity increased considerably from 23.92 to $47.94 \%$ by ca. $100 \%$ after the HIB treatment. It is very interesting that such significant increase in the crystal formation occurred simply through entering the yarn into a bath for a very short time. This can be explained by the fact that the high liquid temperature makes amorphous chains in mobile molten state which with high frictional drag of the bath, provides more possibility for the chains to get oriented and crystallized during the HIB process.

Table 3 also shows the effect of drawing on the crystal formation of control and HIB filaments. As can be seen, the drawing raised the crystallinity in both $\mathrm{HIB}$ and control samples. Such crystal growth probably occurred through the polyester chains in the amorphous region, as the drawing temperature above the $T_{g}\left(70^{\circ} \mathrm{C}\right)$ could make them
Table 4 Molecular orientation of undrawn and drawn control and HIB PTT fibers

\section{SN Applied Sciences}

\begin{tabular}{lllllll}
\hline Sample type & UC-1000 & UC-2000 & UH-1000 & UH4-2000 & DC-2000 & DH4-2000 \\
\hline Birefringence & 0.014 & 0.039 & 0.062 & 0.065 & 0.064 & 0.066 \\
\hline
\end{tabular}


Fig. 7 2D WAXS photographs of undrawn control (a), undrawn HIB (b), c drawn control $(D R=1.40)$, and d drawn PTT $(D R=1.11)$ yarns

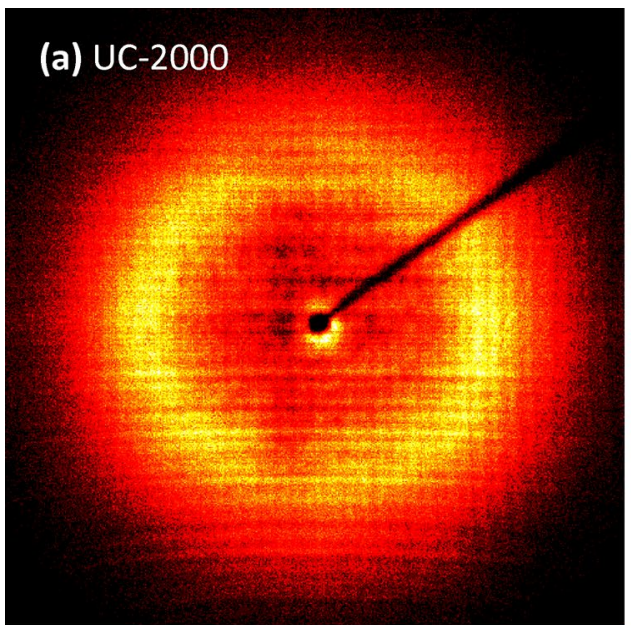

\section{(b) UH4-2000}
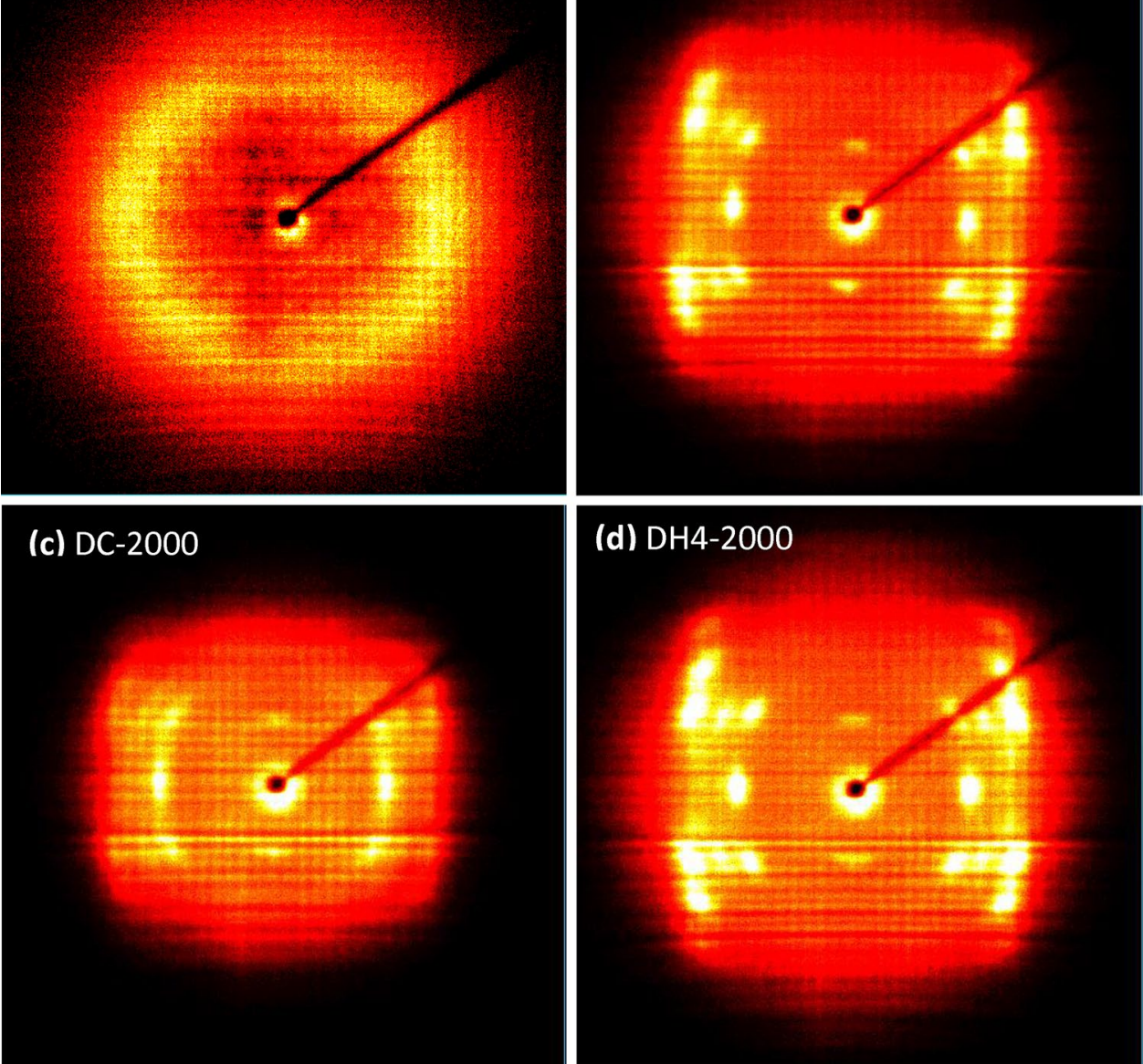

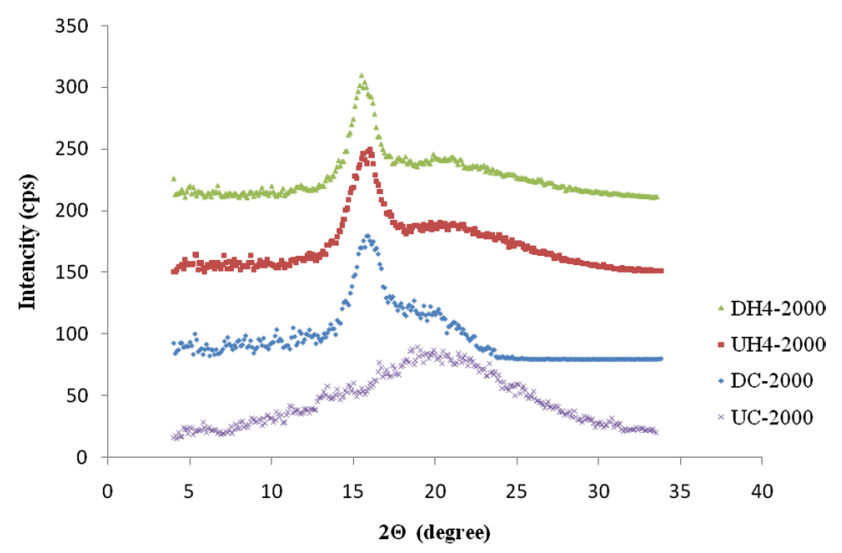

Fig. 8 Equatorial scans of undrawn and drawn control and HIB PTT yarns

mobile enough to be crystallized in the fiber. The obtained high crystallinity (50\%) contributes to the high tenacity of the drawn HIB sample.

\subsubsection{Molecular orientation}

One of the important aspects of HIB treatment is to increase the molecular orientation during fiber formation process. In conventional melt spinning, PTT filaments generally have a low molecular orientation, as the rapid crystal formation restrains the chain mobility for further orientation which affects the mechanical properties of the fibers. This problem can be addressed well in HIB process as the liquid at a high temperature $\left(>T_{g}\right)$ can increase chain mobility for more molecular orientation and crystal formation under the high drag of the liquid bath.

The molecular orientation of the samples was investigated by measuring birefringence. Table 4 shows the birefringence results of undrawn and drawn control and HIB PTT samples at different take-up speeds. As seen in this table, higher take-up speed increased the molecular orientation in undrawn control and HIB samples due to the higher threadline stress on the chains. More importantly, the HIB samples have considerably higher birefringence than control fibers. For example, at $2000 \mathrm{~m} / \mathrm{min}$ the liquid bath treatment increased the fiber orientation from 0.039 


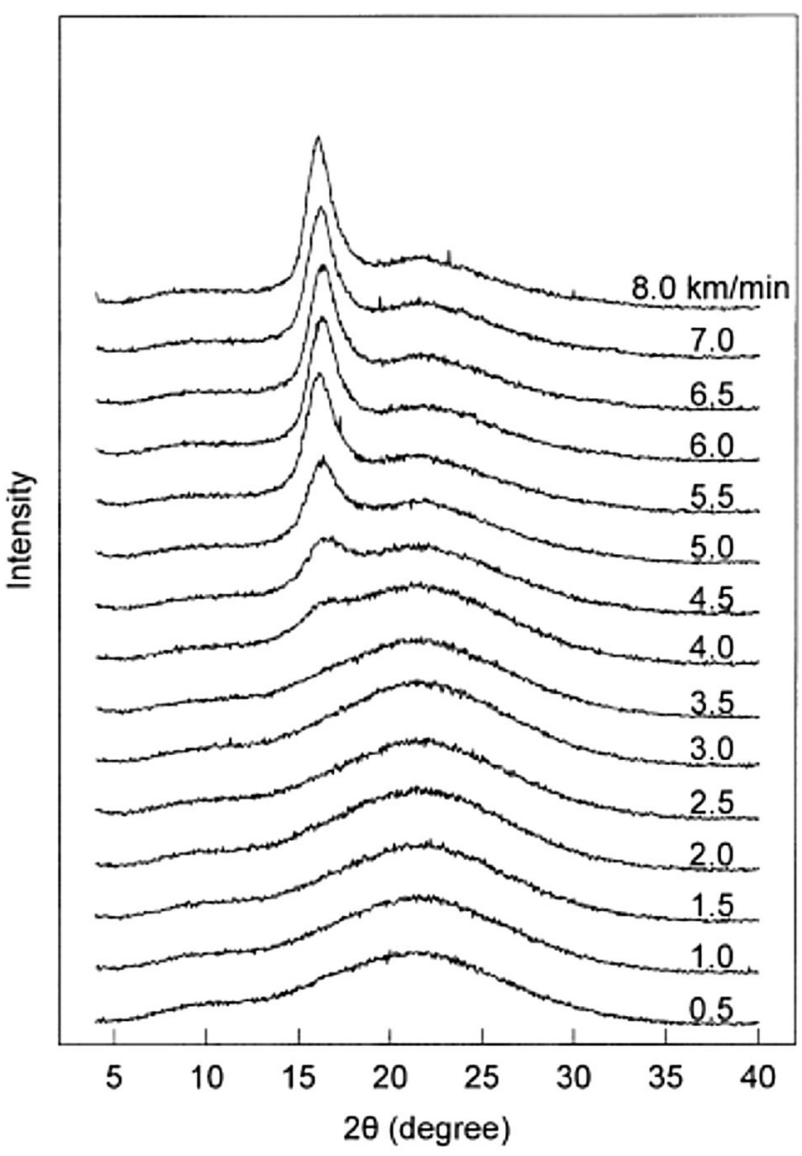

Fig. 9 Equatorial scans of as-spun PTT fibers at different spinning speeds [9]

to 0.065 by ca. $66.67 \%$. The reason is related to the high frictional drag and high liquid temperature of the bath, which increased the mobility of the polyester chains to be extended and aligned along the fiber axis. Also, the table shows that drawing of the as-spun yarns resulted in higher molecular orientation in both control $(0.064)$ and HIB fiber (0.066) (Table 4). As the drawing temperature is higher than the polymer $\mathrm{T}_{\mathrm{g}}$, the amorphous chains are sufficiently mobile to become more oriented in the fiber under DR of 1.11 .

\subsubsection{Crystalline structure}

In order to understand the effect of HIB process on the tensile performance of PTT filaments, their crystal structures need to be known well. Only one crystal form has been known for PTT. The unit cell has a triclinic structure with $a=4.637 \AA, b=6.226 \AA, c=18.64 \AA$ (fiber axis), $a=98.4^{\circ}$, $\beta=93.0^{\circ}$, and $\gamma=111.5^{\circ}$, and a density of $1.432 \mathrm{~g} / \mathrm{cm}^{3}$. Each cell contains a single chain with two repeat monomer units [7].

Figure 7 displays 2D WAXS patterns of undrawn and drawn control and HIB PTT yarns. The typical diffuse amorphous halo of the undrawn control sample can be clearly seen (Fig. 7a). The halo can also be related to existence of small and imperfect crystals, which are difficult to be detected by XRD. Such imperfect crystals were more developed during the drawing process and resulted in an arc pattern in the X-ray plot as seen in Fig. 7c. The diffraction arc also appeared in the $\mathrm{X}$-ray pattern after liquid bath treatment, indicating the existence of crystalline structures in the HIB fiber (Fig. 7b). The formation of such crystals is related to the high drag and high temperature of the liquid bath, which provide enough chain mobility and tension for the polyester chains to be folded and crystallized inside the filaments. Further drawing (DR=1.11) HIB fibers developed more the crystalline structures and resulted in thicker arc pattern as seen in Fig. $7 \mathrm{~d}$.

Figure 8 shows the equatorial $X$-ray diffraction profiles of undrawn and drawn HIB and control PTT yarns. As it can be observed, the undrawn control (UC-2000) has an amorphous halo appearing near $2 \theta=21^{\circ}$, whereas the drawn control (DC-2000) and the undrawn/drawn HIB fibers (UH4-2000 and DH4-2000) have a crystalline peak centers at ca. $2 \theta=16.5^{\circ}$. Such reflection index is related to (010) plane of PTT crystals. When these results compared with former PTT fiber studies (Fig. 9), we can say the crystal structure of the undrawn HIB filament at $2000 \mathrm{~m} / \mathrm{min}$ is comparable with conventionally melt-spun fibers at very high speeds of $7000-8000 \mathrm{~m} / \mathrm{min}$. Such comparison implies that instead of using expensive high-tech winders in the spin-line for PTT yarns, a liquid bath can simply be utilized with low-speed winders and fibers with

Table 5 Tensile and structural properties of undrawn and drawn HIB $(D R=1.11)$ and control PTT yarns

\begin{tabular}{|c|c|c|c|c|c|c|}
\hline Undrawn samples & $\begin{array}{l}\text { HIB conditions (cm, } \\
\left.{ }^{\circ} \mathrm{C}, \mathrm{m} / \mathrm{min}\right)\end{array}$ & Tenacity $(\mathrm{g} / \mathrm{d})$ & Strain (\%) & Deg. of cryst. (\%) & Crystal Size $(\AA ̊)$ & $\Delta \mathrm{n}$ \\
\hline UC-2000 &,,-- 2000 & $1.79 \pm 0.19$ & $233.65 \pm 24.62$ & 23.9 & - & 0.039 \\
\hline DC-2000 &,,-- 2000 & $2.82 \pm 0.33$ & $100.87 \pm 11.14$ & 44.92 & 44.80 & 0.064 \\
\hline UH4-2000 & $18 / 88 / 2000$ & $4.04 \pm 0.23$ & $85.09 \pm 6.25$ & 47.94 & 37.67 & 0.065 \\
\hline DH4-2000 & $18 / 88 / 2000$ & $4.76 \pm 0.28$ & $51.76 \pm 5.32$ & 50.06 & 49.02 & 0.066 \\
\hline
\end{tabular}



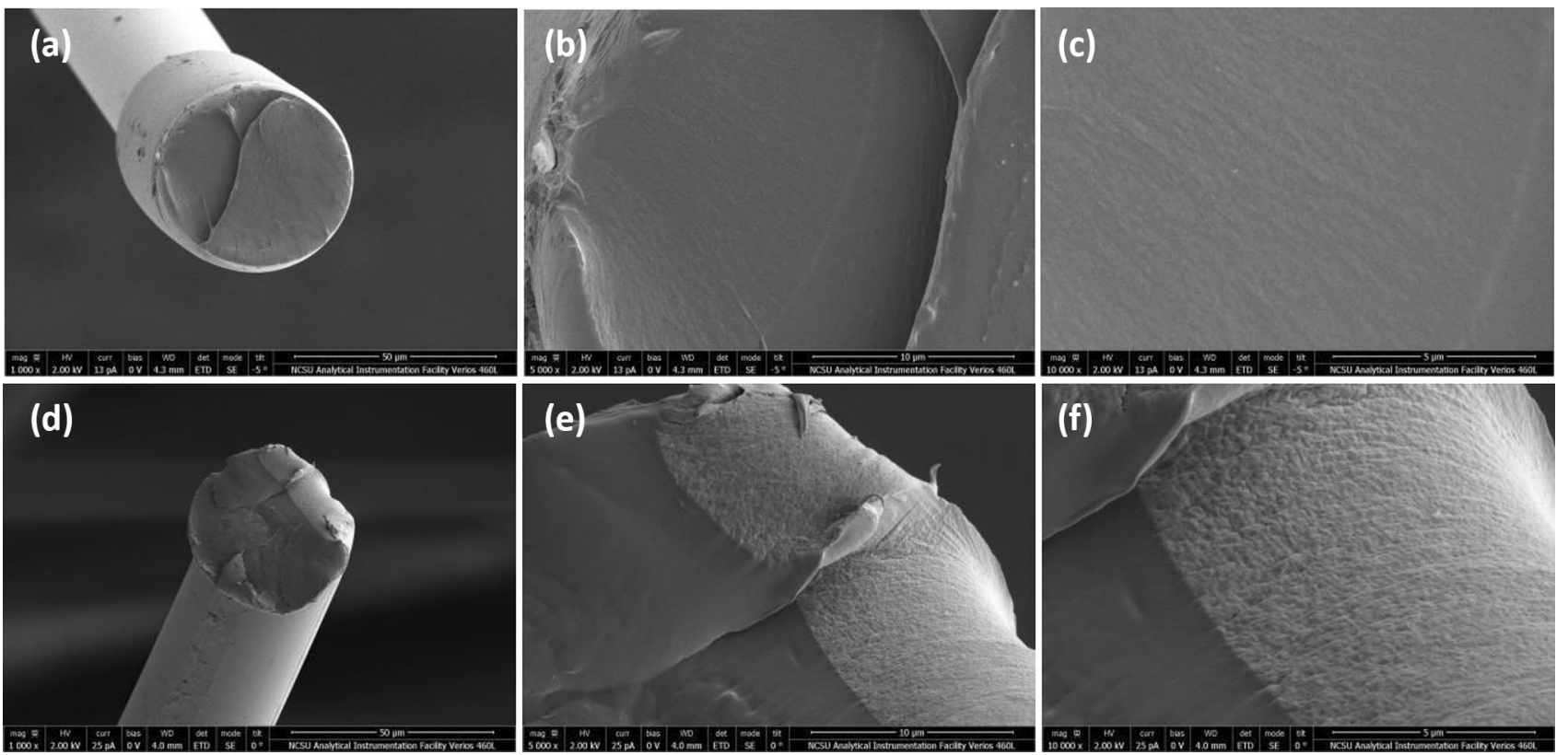

Fig. 10 Cross sectional view of undrawn control (UC-2000) (top) and undrawn HIB (UH4-2000) (down) at three magnifications (left: 1000 $\times$, $50 \mu \mathrm{m}$ scale; middle: $5000 \times ; 10 \mu \mathrm{m}$ scale, right: $10,000 \times 5 \mu \mathrm{m}$ scale)

Fig. 11 Microscopy images of drawn untreated (DC-2000) and treated (DH4-2000) PTT filaments
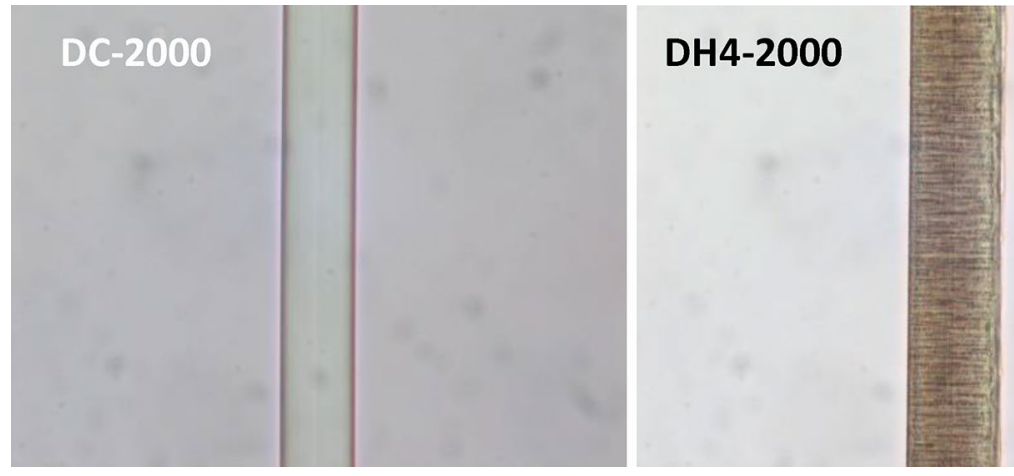

desirable morphology and higher tensile performance can be produced.

Figure 8 also displays the influence of drawing on the crystalline structures of PTT fibers. The appearance of a halo near the crystalline peak is related to oriented amorphous phase in the samples. Like other semi-crystalline polymers (e.g. PA-6 [29], PET [30]), PTT has two noncrystalline regions: oriented amorphous and unoriented amorphous phase [8]. In the oriented region, the macromolecules are preferentially oriented along fiber axis and they can contribute to high tensile performance. As Fig. 8 shows the effect of drawing on the oriented amorphous halo of the control and HIB fibers is not the same. In fact, the drawn control fiber (DC-2000) has smaller oriented amorphous halo/phase, indicating that the drawing transforms part of the oriented amorphous phase into crystalline region. By comparison, for the HIB sample (DH-2000) the drawing did not affect the area of the amorphous halo, implying that the new crystal formation is more likely happened through the transformation of unoriented amorphous region in the fiber. The crystal size of the control and HIB samples are shown in Table 5 . As can be seen, the drawing increased the crystal size of HIB fiber from 37.67 (for UH4-2000) to $49.02 \AA$ (for DH4-2000) by ca. $30 \%$. Higher crystallinity, larger crystal size, and more oriented amorphous region lead the drawn HIB fiber to have higher tenacity and lower strain than the drawn control filament.

\subsubsection{Fiber morphology}

Fiber performance is affected by the morphology and fine structure of the filament. To understand the influence of HIB treatment on fibers morphology, their inside structure was investigated by SEM. Figure 10 displays the cross 
sectional view of the undrawn fibers before and after HIB process. As it can be seen, the control sample has a smooth morphology whereas the HIB sample has a compact fibrillar precursor. The morphology of the drawn fibers was also examined using optical microscopy. Figure 11 shows the plain views of the control and HIB filaments after drawing. The appearance of the kink band structure on the drawn HIB fibers can be clearly seen. Lyoo et al. [4] observed similar kinks in the cold drawing of PTT fibers. They attributed such banded structure to a preferential slip plane parallel to the lamella inside the filaments. The banded morphology indicates that some PTT chains have difficulty in moving probably due to the low temperature used for drawing. It is generally said that the formation of kink structures is closely related to the fibrillar morphology in a fiber $[4,31]$. The fibrillar precursor was also appeared inside HIB fibers for other polymers (e.g. PA-6 [22], PP [12], and PET [32]). Such fibrillar structure can create substantial surface area for the load transfer from the surface to the center of fibers, it can contribute considerably to the tensile properties of the yarn.

\section{Conclusion}

The process of melt spinning PTT was modified by the inclusion of horizontal isothermal bath (HIB) in the spinning line. It was found that the HIB process can improve considerably the structural and mechanical properties of PTT fibers. In fact, the bath increased the tensile strength up to $4.04 \pm 0.23$ by ca. $125.7 \%$ and reduced the tensile strain down to $85.09 \pm 6.25 \%$ by ca. $174.6 \%$. Such superior tenacity was obtained at a low take up speed $(2000 \mathrm{~m} /$ $\mathrm{min}$ ) and simply through entering the threadline into the bath for just some milliseconds, which otherwise needs much higher take-up speeds ( $8000 \mathrm{~m} / \mathrm{min}$ ) in traditional melt spinning. Also, the results showed that the liquid bath increased birefringence from 0.039 to 0.066 , by ca. $70 \%$ and the crystallinity from 23.9 to $47.94 \%$ by $100 \%$. Drawing the HIB fiber with a low DR of 1.11 raised the tenacity up to $4.76 \pm 0.28$ and reduced the stain down to $51.76 \pm 5.32 \%$. The combined effects of high crystallinity, large crystal size, high molecular orientation, and compact fibrillar morphology contributed to such the high tensile strength. The obtained tenacity is greater than the maximum strength of $3.3 \mathrm{~g} / \mathrm{d}$ reported before.

Acknowledgements Mesbah Najafi acknowledges Birgit Anderson, Teresa White, and Judy Elson in the Wilson College of Textiles, and Chuck Mooney and Ching-Chang Chung in Analytical Instrumentation Facility of NCSU for their assistance.

\section{Compliance with ethical standards}

Conflict of interest The authors declare that they do not have conflict of interest.

\section{References}

1. Kim JH, Yang SS, Hudson SM (2011) Comparison of the structureproperty relationships for PTT and PET fibers spun at various take-up speeds. Fibers Polym 12:771-777

2. Vasanthan N, Manne NJ, Krishnama A (2013) Effect of molecular orientation on the cold crystallization of amorphous-crystallizable polymers: the case of poly(trimethylene terephthalate). Ind Eng Chem Res 52:17920-17926

3. Yamen M, Ozkaya S, Vasanthan N (2008) Structural and conformational changes during thermally-induced crystallization of poly(trimethylene terephthalate) by infrared spectroscopy. J Polym Sci Part B Polym Phys 46:1497-1504

4. Lyoo WS, Lee HS, Ji BC, Han SS, Koo K, Kim SS, Kim JH, Lee JS, Son TW, Yoon WS (2001) Effect of zone drawing on the structure and properties of melt-spun poly(trimethylene terephthalate) fiber. J Appl Polym Sci 81:3471-3480

5. Designed for perfromance. Derived from nature 2012. http:// www.dupont.com/content/dam/dupont/products-and-servi ces/fabrics-fibers-and-nonwovens/fibers/documents/2012 SORONA-final.pdf. Accessed 25 Sept 2016

6. Dupont sorona polymer: a new bio based material for 21 st century. http://www2.dupont.com/Hyundai_Kia/en_US/assets/ downloads/presentations/Sorona_Korea_Kurian.pdf. Accessed 25 Sept 2016

7. Wu J, Schultz JM, Samon JM, Pangelinan AB, Chuah HH (2001) In situ study of structure development in poly (trimethylene terephthalate) fibers during stretching by simultaneous synchrotron small- and wide-angle X-ray scattering. Polym J 42:7141-7151

8. Wu J, Schultz JM, Samon JM, Pangelinan AB, Chuah HH (2001) In situ study of structure development during continuous hotdrawing of poly(trimethylene terephthalate) fibers by simultaneous synchrotron small- and wide-angle X-ray scattering. Polym J 42:7161-7170

9. Wu G, Li H, Wu Y, Cuculo JA (2002) Structure and property studies of poly(trimethylene terephthalate) high-speed melt spun fibers. Polym J 43:4915-4922

10. Chuah HH (2015) Synthesis, properties and applications of poly (trimethylene terephthalate). Wiley, Hoboken, pp 361-397

11. Avci H, Kotek R, Toliver B (2014) Controlling of threadline dynamics via a novel method to develop ultra-high performance polypropylene filaments. Polym Eng Sci 55:1-13

12. Avci H, Kotek R, Yoon J (2013) Developing an ecologically friendly isothermal bath to obtain a new class high-tenacity and high-modulus polypropylene fibers. J Mater Sci 48:7791-7804

13. Chen P, Afshari M, Cuculo JA, Kotek R (2009) Direct formation and characterization of a unique precursor morphology in the melt-spinning of polyesters. Macromolecules 42:5437-5441

14. Miyata K, Ito H, Kikutani T, Okui N (1998) Effect of liquid isothermal bath in high-speed melt spinning of poly (ethylene 2, 6-naphthalene dicarboxylate). Sen'i Gakkaishi 54:661-671

15. Chen J-Y, Tucker PA, Cuculo JA (1997) High-performance PET fibers via liquid isothermal bath high-speed spinning: Fiber properties and structure resulting from threadline modification and posttreatment. J Appl Polym Sci 66:2441-2455 
16. Kikutani T, Sato M, Radhakrishnan J, Okui N, Takaku A (1996) High melt spinning of PET via LIB machanism, simulation, 1996. Int Polym Process 11:42-49

17. Wu G, Tucker PA, Cuculo JA (1997) High performance PET fibre properties achieved at high speed using a combination of threadline modification and traditional post treatment. Polym J 38:1091-1100

18. Hotter J, Cuculo J (1998) Effect of liquid isothermal bath position in modified poly (ethylene terephthalate) PET melt spinning process on properties and structure of As-spun and annealed filaments. J Appl Polym Sci 69:2051-2068

19. Wu G, Jiang J, Tucker P, Cuculo J (1996) Oriented noncrystalline structure in PET fibers prepared with threadline modification process. J Polym Sci Polym Phys 34:2035-2047

20. Huang B, Tucker P, Cuculo J (1997) High performance poly (ethylene terephthalate) fibre properties achieved via high speed spinning with a modified liquid isothermal bath process. Polym J 38:1101-1110

21. Wu G, Zhou JACQ, Chen J, Hotter JF, Tucker PA (1995) The effect of a liquid isothermal bath in the threadline on the structure and properties of poly (ethylene terephthalate) fibers. J Appl Polym Sci 55:1275-1289

22. Kotek R, Najafi M, Avci H (2015) High-performance filaments by melt spinning low viscosity nylon 6 using horizontal isothermal bath process. Polym Eng Sci 47:21-25

23. Najafi M, Kotek R (2014) Unique polyamide fibers by hIB process. In: The fiber society technical conference, Liberec, Czech Republic

24. Najafi M, Nasri L, Kotek R (2016) High-performance nylon fibers. In: Bhat G (ed) Structure and properties of high-performance fibers. Woodhead Publishing, Sawston

25. Avci H, Najafi M, Kilic A, Kotek R (2015) Highly crystalline and oriented high-strength poly(ethylene terephthalate) fibers by using low molecular weight polymer. J Appl Polym Sci 42747:1-15

26. Kotek R, Jung W, Smith B (2001) Effect of high speed spinning on physical properties and dyeing kinetics of PTT filaments. Chem Fibers Int 51:204-206

27. Vasanthan N, Manne NJ (2013) Strain-induced crystallization and conformational transition of poly(trimethylene terephthalate) films during uniaxial deformation probed by polarized infrared spectroscopy. Ind Eng Chem Res 52:12596-12603

28. Chuah HH (2001) Orientation and structure development in poly(trimethylene terephthalate) tensile drawing. Macromolecules 34:6985-6993

29. Murthy NS, Minor H, Bednarczyk C, Krimm S (1993) Structure of the amorphous phase in oriented polymers. Macromolecules 26:1712-1721

30. Wu G, Jiang J-D, Tucker PA, Cuculo JA (1996) Oriented noncrystalline structure in PET fibers prepared with threadline modification process. J Polym Sci Part B Polym Phys 34:2035-2047

31. Polis DL, Winey KI (1998) Controlling kink band morphology in block copolymers: threshold criteria and stability. Macromolecules 31:3617-3625

32. Yoon JH, Avci H, Najafi M, Nasri L, Hudson SM, Kotek R (2016) Development of high-tenacity, high-modulus poly(ethylene terephthalate) filaments via a next generation wet-melt-spinning process. Polym Eng Sci. https://doi.org/10.1002/pen.24406

Publisher's Note Springer Nature remains neutral with regard to jurisdictional claims in published maps and institutional affiliations. 